\title{
Low and mid-frequency pulsations in the polar cap: polarization pattern and MLT dependence of the spectral power during the descending phase of the solar cycle
}

\author{
Marcello De Lauretis, Patrizia Francia, Andrea Piancatelli, Massimo Vellante and Umberto Villante \\ Dipartimento di Fisica, Università dell'Aquila, L'Aquila, Italy \\ Consorzio Area di Ricerca in Astrogeofisica, L'Aquila, Italy
}

\begin{abstract}
We present a statistical analysis of ULF (1-100 mHz) geomagnetic measurements conducted during years 20032006 at the Italian/French base of Concordia at Dome C, close to the geomagnetic pole, and at the Italian base «Mario Zucchelli» at Terra Nova Bay, also located in the polar cap, but at lower latitude. Our analysis shows that high latitude ULF pulsation power is largely controlled by the solar wind speed. At Terra Nova Bay the power shows a maximum at local noon, clearly related to cusp and closed field lines phenomena. At few $\mathrm{mHz}$, the polarization pattern indicates field line resonances driven, just equatorward with respect to the station, by waves propagating tailward; the polarization of higher frequency pulsations, mostly originated from interplanetary upstream waves, suggests waves propagating sunward from the night sector. At Dome $\mathrm{C}$ the wave power shows a small enhancement in the local morning, more pronounced for mid-frequency pulsations; the polarization pattern, at all frequencies, appears to indicate waves propagating sunward from the night sector, suggesting a propagation channel to the ground via the magnetotail lobes.
\end{abstract}

Key words ULF waves - Antarctica-Geomagnetic pulsations - Polarization pattern - upstream waves

\section{Introduction}

Geomagnetic field measurements at very high latitudes are important to understand some physical processes controlling the energy transfer from the solar wind (SW) to the magnetosphere. High latitude regions, indeed, lie at foot-

Mailing address: Dr. Marcello De Lauretis, Dipartimento di Fisica, via Vetoio 10, 67010 Coppito-L'Aquila, Italy; e-mail: marcello.delauretis@aquila.infn.it prints of geomagnetic field lines extending to extreme magnetospheric regions (such as the magnetopause, the tail lobes, etc.), where the electromagnetic SW-magnetosphere coupling occurs.

Antarctic arrays provide a unique opportunity to explore the polar cap, cusp and auroral oval, where different physical processes operate. The availability of measurements conducted with similar instrumentation at the Italian geomagnetic station Mario Zucchelli at Terra Nova Bay (TNB, corrected geomagnetic latitude $80^{\circ} \mathrm{S}$ ) and at the Italian-French base of Concordia, at DomeC (DMC, corrected geomagnetic latitude $89^{\circ} \mathrm{S}$ ) allows an interesting comparison between measurements in different regions. Indeed, both stations are located in the polar cap, at the footprints of open field lines, but, due to the Earth's rotation, TNB, which is 
at lower latitude, approaches around noon the cusp and closed field lines.

Several analyses of TNB data, available since 1994, have demonstrated that the polar cap is an active area for ULF wave activity in the low and mid frequency range, from $\mathrm{Pc} 5(1-7 \mathrm{mHz})$ up to Pc4 (7-22 mHz) and Pc3 (22-100 mHz) pulsations (Villante et al., 2000a; 2000b; Francia et $a l ., 2005)$. According to these investigations, it is found that, independently of frequency, the ULF power maximizes around noon, when the station approaches the cusp and closed field lines; moreover, in conflict with simultaneous observations at a cusp station (South Pole, $\sim 74^{\circ} \mathrm{S}$ ), no evidence for substorm related activity is detected in the nighttime sector.

On the other hand at DMC, in the deep polar cap, during a test campaign, De Lauretis et al., (2005) found approximately the same power through the day without any enhancement at local noon. In addition, a comparison with TNB data showed that during disturbed magnetospheric conditions, when the cusp shifts to lower latitudes and TNB is embedded in the polar cap for the whole day, the pulsation activity at the two stations becomes very similar: in particular at both stations the pulsation power appears driven by SW pressure pulses and highly dependent on the SW speed (Villante et al., 2000a; 2000b; De Lauretis et al., 2005).

Significant information on the generation and propagation mechanisms of ULF waves can be obtained from their polarization characteristics. Indeed, in the low-frequency range (Pc4-5), waves generated by the KelvinHelmholtz instability (KHI) at the magnetopause (Atkinson and Watanabe, 1966) are expected to propagate antisunward, i.e. westward in the local morning and eastward in the afternoon. As a consequence, at a southern ground station, pulsations should exhibit a clockwise (CW, looking downward on the Earth) and counterclockwise $(\mathrm{CCW})$ polarization in the morning and afternoon, respectively. However, the polarization pattern can be modified by the resonant coupling between compressional waves and Alfven modes (Southwood, 1974); in particular, for a given frequency, polarization reversals are predicted at the latitude $\Lambda_{1}$ of the resonant field line and at the higher latitude $\Lambda_{2}$ corresponding to the amplitude minimum between the magnetopause and the resonant field line. Both these latitudes have a local time dependence: at high latitudes, $\Lambda_{1}$ is higher around local noon with respect to dawn and dusk, due to the diurnal variation of the field line length in the outer magnetosphere (Mathie et al., 1999). A statistical analysis of low-frequency magnetic fluctuations at TNB (Francia et al., 2005) showed that the polarization characteristics are consistent with a station always located poleward with respect to the latitude of the resonance; in particular, for frequencies below 4 $\mathrm{mHz}$, the pattern (4 sectors of alternate polarization through the day) suggests a station embedded, as it rotates into the sunward magnetosphere, in a region between $\Lambda_{2}$ and $\Lambda_{1}$ where resonance effects can be still observed («resonance region»). Obviously, a similar behavior should progressively disappear with increasing latitude and vanish at DMC.

In the mid-frequency range $(\mathrm{f}=10-100 \mathrm{mHz})$ magnetospheric ULF waves are usually interpreted in terms of penetration of upstream waves generated in the foreshock region by cyclotron resonance with ions reflected off the bow shock along the interplanetary magnetic field (IMF) lines (Greenstadt et al., 1980). The most accepted mechanisms for the entry of upstream wave energy into the magnetosphere are basically two: one suggests a direct transmission from the subsolar bow shock (Russell et al., 1983), while the second suggests an indirect process involving particle and current modulation at the near-cusp ionosphere (Engebretson et al., 1991; Olson and Szuberla, 1997). De Lauretis et al. (2005) observed at DMC a clear dependence of the pulsation frequency on the IMF strength and suggested upstream waves as source of pulsations also in the deep polar cap. The observed frequency was found in some cases to be higher than that predicted assuming upstream wave generation close to the bow shock nose (Takahashi et al., 1984). This might indicate that high-latitude $\mathrm{Pc} 3$ pulsations might be related to waves generated along the flanks of the bow shock where their frequency is expected to be higher than at the nose (De Lauretis et al., 2005). This aspect appears consistent with the results obtained by Chugunova et 
al. $(2003 ; 2004)$ who suggested an additional propagation path of upstream waves to the ground via the magnetotail lobes, causing Pc34 activity in the polar cap, at the footpoint of open field lines stretched into the tail.

This paper presents a statistical analysis of low and mid-frequency pulsations observed at DMC and at TNB during years 2003-2006, in the declining phase of the solar cycle and discuss the magnetic local time (MLT) dependence of the spectral power as well as the aspects of the polarization pattern.

\section{Instrumentation and data analysis}

A similar experimental apparatus operates at TNB and DMC (table I for their coordinates). It consists of a high sensitivity three-axial induction coil (search-coil) and an automatic acquisition system, with timing provided via GPS, that samples the data at a rate of $1 \mathrm{~Hz}$. The two magnetometers have similar characteristics, with an amplitude response linearly increasing in the range $1-100 \mathrm{mHz}$ (about $2-5$ $\mathrm{mV} / \mathrm{nT} / \mathrm{mHz}$ ).

Unfortunately, due to technical problems, simultaneous measurements are not available. In this analysis we use data acquired at TNB through 2003, 2004 and 2006 and at DMC during 2005.

The spectral analysis of the $\mathrm{H}$ and D components was conducted by means of the Welch periodogram method over 1-hour intervals. The intrinsic pre-whitening effect of each searchcoil sensor was compensated in the frequency domain by dividing the power spectra estimates by the square of the amplitude response. Polarization parameters were computed using the technique for partially polarized waves as proposed by Fowler et al. (1967). In particular, the polarization ratio $\mathrm{R}$ (i.e. the ratio between the polarized and total intensity of the horizontal signal) and the ellipticity $\varepsilon$ (i.e., the ratio between the minor and the major axis of the polarization ellipse in the horizontal plane) was evaluated over each hour. In order to consider only intervals characterized by a negligible noise component and by a well identified polarization sense, this analysis was restricted to intervals with $\mathrm{R}>0.7$ and $|\varepsilon|>0.1$.

For a comparison with the interplanetary medium conditions we considered the SW and IMF parameters from the ACE spacecraft at the L1 libration point upstream of Earth.

\section{Observations and experimental results}

Figure 1 shows the MLT dependence of the annual average of the hourly total power $(\mathrm{H}+\mathrm{D})$ for Pc5 (1-7 mHz), Pc4 (7-22 mHz) and Pc3 $(22-100 \mathrm{mHz})$ pulsations. Independently on frequency, at TNB a clear diurnal modulation emerges in each year, with a maximum at noon when the station approaches the cusp and closed field lines. By contrast, at DMC the MLT dependence only shows a small enhancement around 03 MLT (which corresponds approximately to noon in local time). Note, in addition, a progressive decrease approaching solar minimum of the average power (horizontal line) observed at TNB (and not contradicted by

Table I. Coordinates of the recording stations.

\begin{tabular}{cccc}
\hline \hline Station & Geographic coordinates & $\begin{array}{c}\text { Corrected geomagnetic } \\
\text { coordinates }\end{array}$ & $\begin{array}{c}\text { Time of magnetic } \\
\text { local midnight (UT) }\end{array}$ \\
\hline Concordia & $75.10 \mathrm{~S}$ & $88.84 \mathrm{~S}$ & \\
$(\mathrm{DMC})$ & $123.38 \mathrm{E}$ & $55.73 \mathrm{E}$ & \\
Mario Zucchelli & $74.69 \mathrm{~S}$ & $80.01 \mathrm{~S}$ & $08: 11$ \\
(TNB) & $164.12 \mathrm{E}$ & $306.94 \mathrm{E}$ & 0 \\
\hline
\end{tabular}



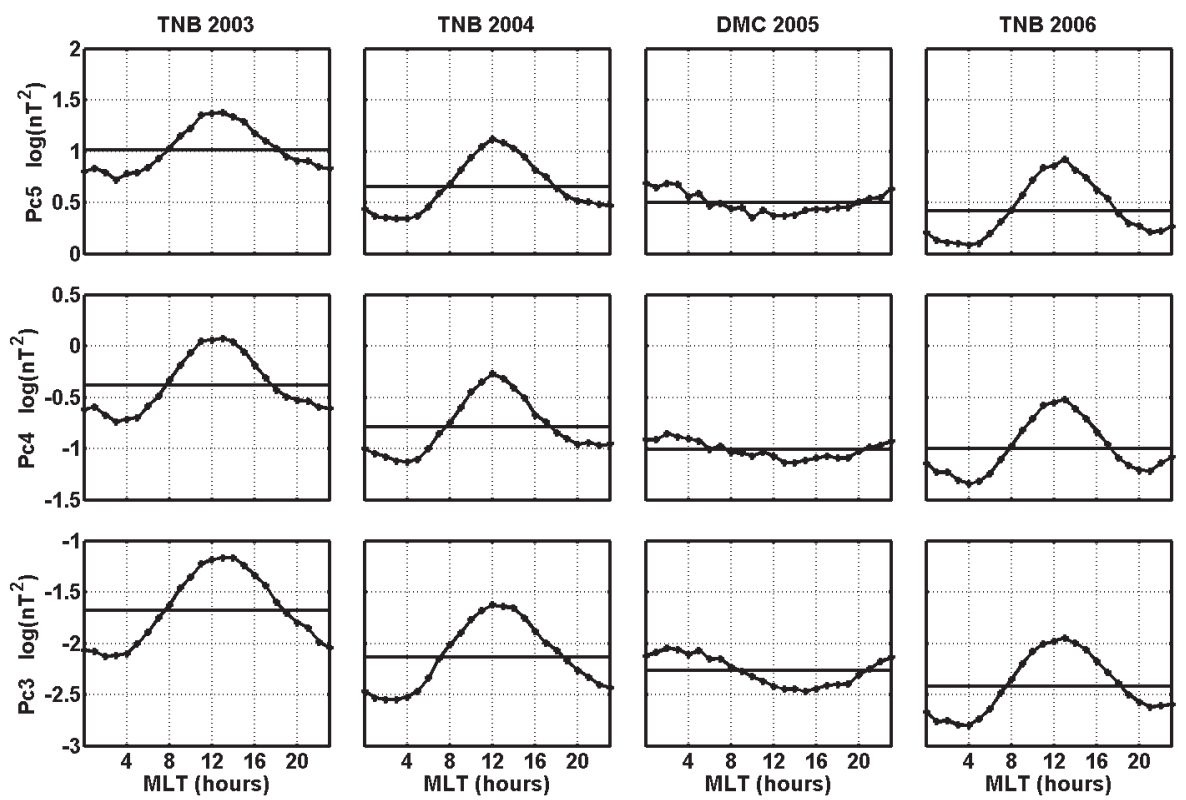

Fig. 1. MLT variation of the hourly power of the horizontal component at TNB and DMC stations through the years. From top to bottom: Pc5 (1-7 mHz), Pc4 (7-22 mHz), Pc3 (22-100 mHz) pulsations.

DMC data): namely the power on average reduces of a factor $\sim 4$ for Pc4-5 pulsations and $\sim 5.5$ for Pc3 pulsations from 2003 to 2006.

In order to investigate the dependence of the ULF power on solar and interplanetary parameters, we performed a correlation analysis. Figure 2 shows, from the top, the logarithm of the monthly averages of power in the whole Pc3-5 frequency band, the sunspot number $(\mathrm{R})$, the amplitude of the IMF $\left(\mathrm{B}_{\mathrm{IMF}}\right)$ and the SW velocity $\left(\mathrm{V}_{\mathrm{SW}}\right)$. All these parameters tend to decrease approaching the solar cycle minimum. The correlation analysis shows that the ULF power is highly correlated both with $\mathrm{B}_{\mathrm{IMF}}(\mathrm{r}=0.79)$ and $\mathrm{V}_{\mathrm{SW}}(\mathrm{r}=0.73)$ suggesting that, at least for monthly scales, the high latitude ULF power is largely controlled by the SW conditions; on the other hand, a high correlation is observed $(\mathrm{r}=0.72)$ between $\mathrm{B}_{\mathrm{IMF}}$ and $\mathrm{V}_{\mathrm{SW}}$.

There also emerges, in particular during 2004 and 2006, a tendency of the ULF power to be lower during local winter, indicating that ionospheric conditions are important at such high latitudes. It is also interesting to note that the annual variation is almost absent during 2003, when $V_{\text {SW }}$ was higher than $500 \mathrm{~km} / \mathrm{s}$ through the whole year reaching the highest values just during local winter. These results are also confirmed by a separate analysis in the three different frequency bands.

Figure 3 shows the 24-hour running averages of Pc3-5 power, $\mathrm{B}_{\mathrm{IMF}}$ and $\mathrm{V}_{\mathrm{SW}}$. In addition to the annual variation already discussed, we find a clear 27-day modulation of the power during 2006, in correspondence with the corotating fast streams which characterize the descending phase of the solar cycle (Tsurutani et al., 1995 and references therein). So, the SW speed dependence is confirmed also for daily scales.

An analysis of the polarization pattern was conducted both at TNB and DMC. Figure 4 we show, in a colour scale, the MLT and frequency dependence of the percentage of CW polarized pulsations through the solar cycle. At TNB, at frequencies of few $\mathrm{mHz}$, the pattern shows almost the same characteristics in each year. In particular, up to $\mathrm{f} \sim 6 \mathrm{mHz}$, the day is divided into 4 sectors with alternate polarization as ex- 


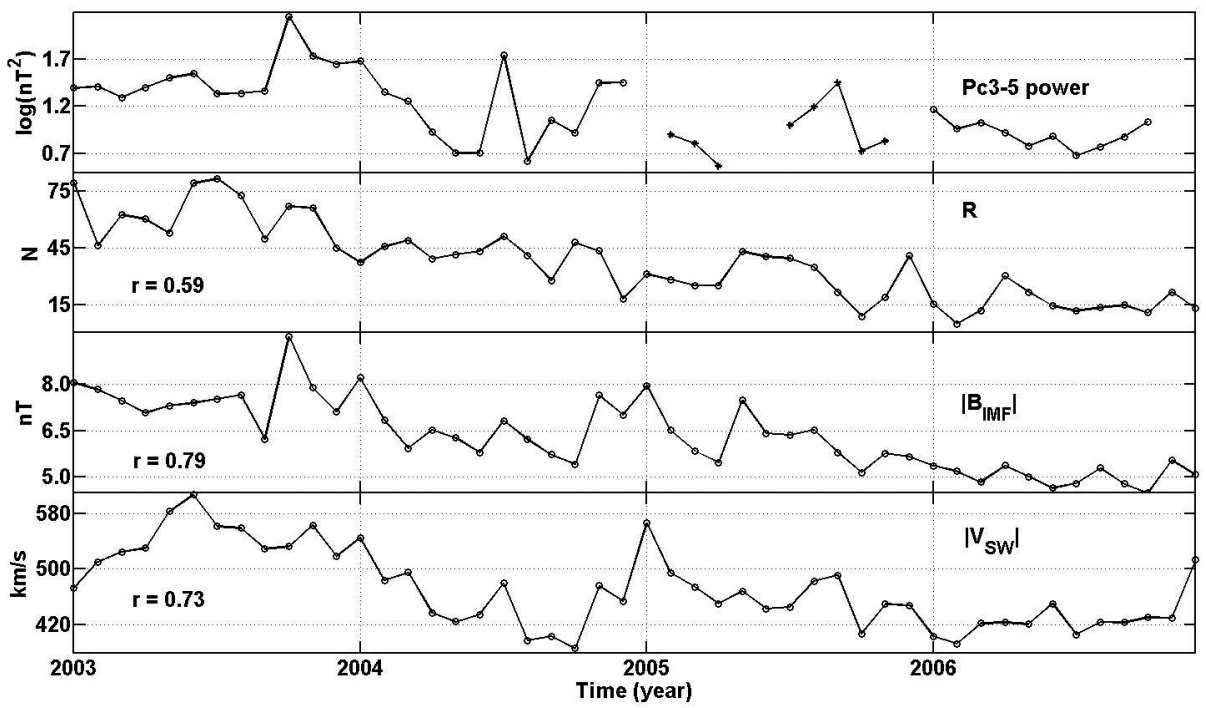

Fig. 2. Monthly averages of ULF power and interplanetary parameters. From top to bottom: Pc3-5 power of the horizontal component, sunspot's number, IMF amplitude and SW speed.

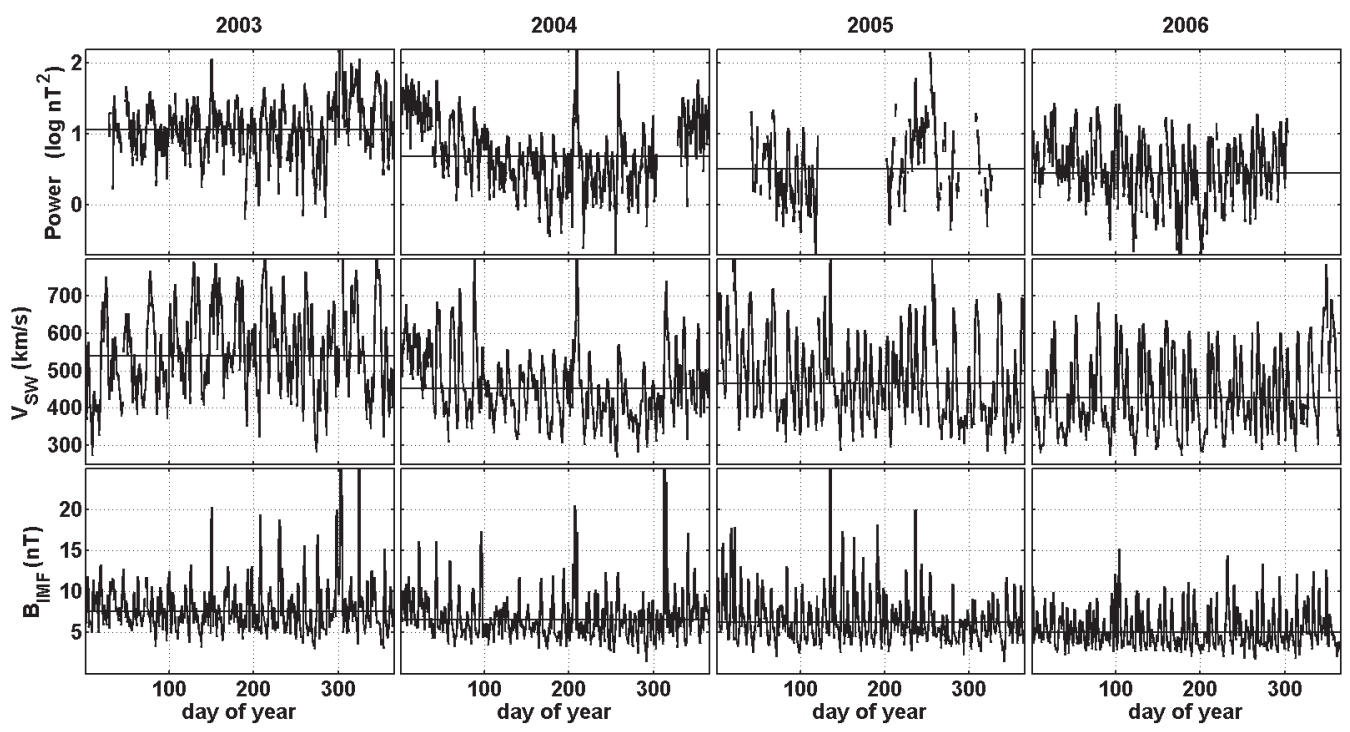

Fig. 3. One-day running averages of Pc3-5 power of the horizontal component at TNB $(2003,2004,2006)$ and DMC (2005) stations, SW speed and IMF amplitude.

pected for waves propagating antisunward (such as those generated by the KHI) when resonance effects are taken into account. Indeed,
TNB is always located poleward with respect to resonant field lines and rotates into the resonance region during daytime hours. At higher 

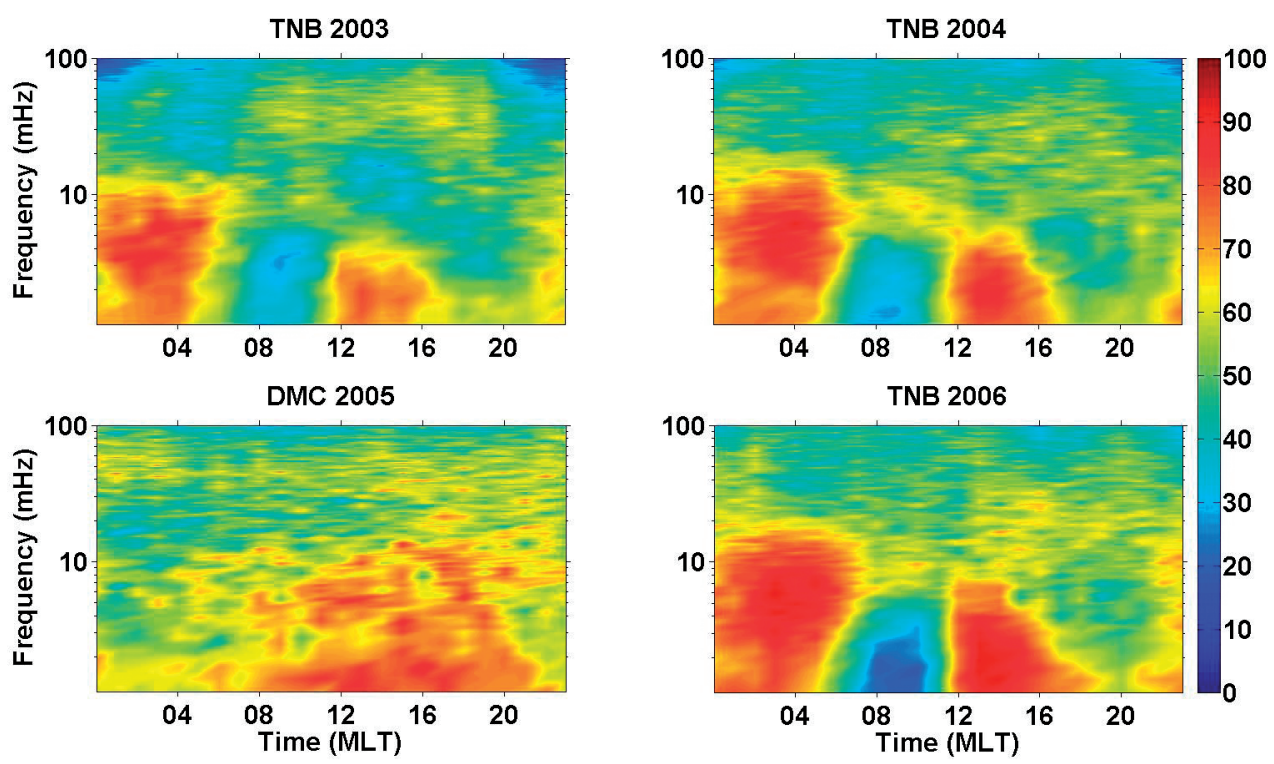

Fig. 4. MLT and frequency distribution of the percentage of CW events at TNB and DMC stations.

frequencies the pattern appears well definite only during 2003, possibly due to the more favourable SW conditions and consequent higher ULF activity. In the $\sim 6-15 \mathrm{mHz}$ frequency range, $\mathrm{CW}$ and $\mathrm{CCW}$ polarizations tend to prevail in the morning and afternoon sectors, as expected far from the resonance for antisunward propagation. Above $\sim 15 \mathrm{mHz}$, the polarization sense is mixed/CW between 08-20 MLT suggesting waves propagating from opposite directions; after 20 MLT the polarization sense remains mostly CCW up to 08 MLT indicating an antisunward propagation in the premidnight sector and sunward in the postmidnight and morning sectors. Note that between 02-04 MLT a mixed structure suggests the presence of waves from opposite directions. In this frequency range, pulsations are currently believed to originate from upstream waves transmitted in the magnetosphere directly from the subsolar bow shock or through the cusp: in this case, the source should be located around noon. Recently, however, an additional path via the magnetotail lobes has been suggested by Chugunova et al. (2003; 2004). Our results seem to confirm their suggestion since the observed polarization pattern indicates sunward propagation from sources in the night sector. During the years 2004 and 2006 the polarization pattern for frequencies higher than $\sim 6 \mathrm{mHz}$ is not well defined; note that during 2004 the mixed/CW structure between 08-20 MLT seems to shift toward lower frequencies, possibly due to the decrease of the IMF amplitude (fig. 2) and the corresponding upstream wave frequency $\left(\mathrm{f}[\mathrm{mHz}] \sim 6 \cdot \mathrm{B}_{\mathrm{IMF}}[\mathrm{nT}]\right.$, Troitskaya and Bolshakova, 1988).

At DMC, up to $10 \mathrm{mHz}$ it emerges a CW polarization between 08-20 MLT and a mixed structure in the nighttime and early morning. At higher frequencies the pattern is mixed, with a slightly predominant $\mathrm{CCW}$ and $\mathrm{CW}$ polarization sense in the morning and in the afternoon, respectively. Generally, the polarization pattern appears to be consistent with waves propagating sunward from a source in the night sector.

\section{Summary and conclusions}

We analyzed the spectral power and the polarization pattern of low and mid-frequency (1- 
$100 \mathrm{mHz}$ ) pulsations at two polar cap stations during the descending phase of the solar cycle.

At TNB the ULF wave power and polarization are controlled by the position of the station which approaches closed field lines in the daytime hours and the cusp at noon. So, the power at both low and mid frequencies is characterized by a broad maximum around noon, which is clearly related to cusp and closed field lines phenomena. At few $\mathrm{mHz}$, the polarization pattern indicates field line resonances driven, just equatorward with respect to the station, by waves propagating tailward, as expected for waves generated by the KHI. On the other hand, mid-frequency signals exhibit a polarization pattern which is indicative of waves propagating sunward from an additional source possibly located in the night sector. This result is in agreement with the conclusions of Chugunova et al. $(2003$; 2004) who proposed an additional propagation path via the magnetotail lobes for Pc3-4 pulsations related to upstream waves.

At DMC, the ULF wave power shows evidence for an enhancement between 01-04 MLT, more pronounced in the Pc3 band. This feature was observed for Pc3-4 pulsations also by Chugunova et al. $(2003 ; 2004)$ at $\mathrm{P} 5\left(87^{\circ} \mathrm{S}\right)$ and can be interpreted in terms of upstream waves which are generated preferentially on the morning side. On the other hand, such enhancement, which occurs approximately around noon in local time, might also reflect ionospheric effects. Note that a similar effect was already observed at DMC for Pc5 pulsations by Lepidi et al. (2003). The polarization pattern is less clear than at TNB; at all frequencies, however, it appears to indicate waves mostly associated with magnetotail processes.

Finally, our analysis shows a correspondence of ULF power and SW parameters and confirms previous results related to the SW speed control of the pulsation power (Villante et al., 2000b; De Lauretis et al., 2005). Indeed, the close relationship between the pulsation power and the SW speed is generally expected since the SW provides the energy for magnetospheric phenomena. On the other hand, the high correlation with IMF strength could be explained considering that simultaneous high values of $\mathrm{V}_{\mathrm{SW}}$ and $\mathrm{B}_{\mathrm{IMF}}$ occur in the interplanetary magnetic clouds.

An additional result of our analysis is represented by the annual modulation of the pulsation power that suggests a significant role of ionospheric conditions in determining the level of the pulsation activity during usual SW speed conditions.

\section{Acknowledgements}

The authors thank N.F. Ness for the ACE MFI data and D.J. McComas for the ACE SWE data.

\section{REFERENCES}

AtKinson, G. and T. Watanabe (1966): Surface waves in the magnetospheric boundary as a possible origin of long period geomagnetic micropulsations, Earth Planet. Sci. Lett., 1, 89-91.

Chugunova, O.M., V.A. Pilipenko and M.J. Engebretson (2003): Statistical features of Pc3-4 pulsations at very high latitudes, Proc. XXVI Annual Seminar, Apatity, (Russia), 103-106.

Chugunova, O.M., V.A. Pilipenko and M. Engebretson (2004): Appearance of Quasi-Monochromatic Pc3-4 Pulsations in the Polar Cap, Geomagn. Aeron., 44, 42-48.

De Lauretis, M., P. Francia, M. Vellante, A. Piancatelli, U. Villante and D. Di Memmo (2005): ULF geomagnetic pulsations in the southern polar cap: simultaneous measurements near the cusp and the geomagnetic pole., J. Geophys. Res., 110, doi: 10.1029/2005JA011058.

Engebretson M.J., L.J. Cahill Jr., R.L. Arnoldy, B.J. Anderson, T.J. Rosenberg, D.L. CARPenter, U.S. INAN and R.H. EATHER (1991): The role of the ionosphere in coupling upstream ULF wave power into the dayside magnetosphere, J. Geophys. Res., 96, 15271542.

Fowler, R.A., B.J. KoticK and R.D. ElLIOTT (1967): Polarization analysis of natural and artificially induced geomagnetic micropulsation, J. Geophys. Res., 72, 28712883.

Francia, P., L.J. LANZERotti, U. Villante, S. LePidi and D. Di Memmo (2005): A statistical analysis of low frequency magnetic pulsations at South Pole, J. Geophys. Res., 110, doi: 10.1029/2004JA010680.

Greenstadt, E.W., R.L. McPherron and K. TAKahashi (1980): Solar wind control of daytime, medperiod geomagnetic pulsations, J. Geomagn. Geoelectr., 32 (suppl. 11), 89 .

Lepidi, S., L. Cafarella, P. Francia, A. Meloni, P. PAlangio and J.J. SchotT (2003): Low frequency geomagnetic field variations at Dome C (Antarctica), Ann. Geophys., 21, 1-10.

MAthie, R.A., F.W. MEnK, I.R. MAnN and D. ORR (1999): Discrete field line resonances and the Alfven continuum in the outer magnetosphere, Geophys. Res. Lett., 26, 659-662. 
Olson, J.V. and C.A.L. Szuberla (1997): A study of Pc3 coherence at high latitudes, J. Geophys. Res., 102, $11375-11383$.

Russell, C.T., J.G. LuhmanN, T.J. Odera and W.F. StuarT (1983): The rate of occurrence of dayside Pc3,4 pulsations: the L-value dependence of the IMF cone angle effect, Geophys. Res. Lett., 10, 663-666.

Southwood, D.J. (1974): Some features of field line resonance in the magnetosphere, Planet. Space Sci., 22, 482-491.

TAKAhashi, K., R.L. McPherron and T. Teresawa (1984): Dependence of the spectrum of $\mathrm{Pc} 3-4$ pulsations on the interplanetary magnetic field, J. Geophys. Res., 89, 2780 .
Troitskaya, V.A. and O.V. Bolshakova (1988): Diagnostics of the magnetosphere using multipoint measurements of ULF-waves, Adv. Space Res., 8, 413-425.

Tsurutani, B.T., Gonzalez W.D., GonZalez A.L.C., TAng F., Arballo J.K. and M. OKada (1995): Interplanetary origin of geomagnetic activity in the declining phase of the solar cycle, J. Geophys. Res., 100, 21717-21733.

Villante, U., S. Lepidi, P. Francia, M. Vellante, A. Meloni and P. PAlangio (2000a): UlF fluctuations at Terra Nova Bay (Antarctica), Annali di Geofisica, 43 (2), 217-227.

Villante, U., M. Vellante and G. De Sanctis (2000b): An analysis of Pc3 and Pc4 pulsations at Terra Nova Bay, Ann. Geophys., 18, 1412-1421. 